\title{
Corneal Biomechanical Properties Post-LASEK for the Correction of Myopia
}

\author{
${ }^{1}$ Ramez Barbara, ${ }^{2}$ Ali Nassar, ${ }^{3}$ David Zadok, ${ }^{4}$ Adel Barbara
}

\begin{abstract}
Introduction: Laser surgeries are effective and safe, however, over the years data has accumulated about several complications of the procedures, particularly corneal ectasia. Various studies demonstrated the importance of the front layers of the cornea to keep its structure fixed. In order to correct various degrees of myopia, different thicknesses of tissue have to be removed. Therefore, it is conceivable that the risk of developing ectasia might increase depending on the depth of corneal tissue removed.

In the last few years, the Reichert Ocular Respond Analyzer (ORA) for measuring the biomechanical parameters of the cornea has been introduced. Studies demonstrated a decrease in the corneal biomechanical parameters after Laser Assisted Sub-epithelial Keratomileusis (LASEK) surgery. However, the, relation between the depth of tissue removed and the amount of change in the biomechanical parameters had not been tested yet.
\end{abstract}

Purpose: To evaluate the relationship between corneal biomechanical parameters and corneal tissue ablation thickness.

Methods: Data was retrospectively collected from patient's files that underwent LASEK in private medical center - Hadassah Optimal in Haifa between January 2009 and May 2010. All patients were operated by same surgeon $(A B)$. In our study we included patients that had at least three ORA measurements at three set periods of time; before the operation, up to 3 months postoperative and more than 3 months postoperative. We divided the patients into 3 groups depending on the refractive error and tested the effect of variable factors (Age, Total depth ablated-TDA, percent of total depth ablated-\%TDA, sex, refraction). The data was analyzed by descriptive statistics and student $\mathrm{t}$-test to find the relation between LASEK and the corneal biomechanical parameters.

Results: Ninety-eight patients participated in our study, which included 98 separate eye operations. A statistically significant decrease $(p<0.001$ ) was found in CRF, CH, IOPg after

\footnotetext{
${ }^{1}$ Ophthalmologist, ${ }^{2}$ Intern, ${ }^{3}$ Professor, ${ }^{4}$ Medical Director

${ }^{1}$ Department of Ophthalmology, Hadassah Optimal, Haifa, Israel

${ }^{2}$ Sackler Faculty of Medicine, Tel Aviv University, Israel

${ }^{3}$ Sackler Faculty of Medicine, Department of Ophthalmology Assaf Harofeh Medical Center, Tel Aviv University, Israel

${ }^{4}$ Hadassah Optimal, Haifa; President, Refractive Surgery Society, Israel
}

Corresponding Author: Ramez Barbara, Ophthalmologist Department of Ophthalmology, Tshernihovski St. 29, Haifa 35701, Israel, e-mail: ramezborbara@gmail.com
LASEK. In group 1 (refraction -0.5 to -2.99 ): on $\mathrm{CH}$ - there was a statistically significant $(p<0.05)$ weak-moderate effect of TDA and \%TDA (Pearson coefficient of $0.4,0.39$ respectively), statistically significant $(p<0.05)$ effect of age. On CRF - we found a statistically significant $(p<0.05)$ weak-moderate effect of TDA and \%TDA (Pearson coefficient of 0.36 for both factors), statistically significant $(p<0.05)$ effect of age. In the second group (refraction value -3 to -5.99 ) only on IOPg a statistically significant $(p<0.05)$ weak effect of TDA (Pearson coefficient of 0.33 ) was demonstrated. In the third group, we did not find any effect of the variable factors on the change in biomechanical properties characteristics after LASEK.

Importance: Corneal biomechanical properties influence corneal behavior in certain eye diseases. Moreover, it has been noted that operations and procedures affect these biomechanical properties of the cornea. Hence, it is of great importance to reveal factors that could affect and change such parameters.

Keywords: LASEK, Corneal biomechanics, Corneal hysteresis, Corneal resistance factor, Pachymetry, Corneal thickness, Intraocular pressure, Laser, Cornea.

How to cite this article: Barbara R, Nassar A, Zadok D, Barbara A. Corneal Biomechanical Properties Post-LASEK for the Correction of Myopia. Int J Kerat Ect Cor Dis 2014;3(1):23-28.

Source of support: Nil

Conflict of interest: None

\section{INTRODUCTION}

Myopia is the most common cause of refractive errors worldwide. In Asia, the prevalence is as high as $70 \%$, in the United States and Europe 30\% and in Africa 10\%. Epidemiological studies have demonstrated genetic, environmental, age and ethnic origin as contributing factors to its development. $^{1}$

Laser corrective eye surgery is very popular nowadays and more than 1 million procedures are performed in the United States alone. ${ }^{2}$ Newer lasers with less than a $1 \mathrm{~mm}$ laser beam and eye tracking systems resulted in smoother ablation surfaces, hence, enhancing wound healing, reducing pain and lowering the risk of stromal haze. Refractive surgery results in corneal biomechanical changes related to reduced corneal thickness, changes in corneal shape and collagen layer composition. ${ }^{3-5}$

Ocular response analyzer (ORA) uses an air puff system that is directed to the eye for $20 \mathrm{~ms} .{ }^{6,7}$ This accurately measured air stream results in changes in corneal shape; the 
corneal flattens (applanation) before becoming concave due to air pressure. A few milliseconds following the first applanation point the air pressure ceases gradually resulting in bouncing back of the cornea to a second applanation point on its way to taking back its original concave shape. Throughout the test, corneal movement features described above are monitored through an electro-optic laser beam. This technique enables the ORA to measure the corneal biomechanical properties based on corneal elastic properties. ${ }^{9}$ Had the cornea been an elastic organ only, then we would expect it to have two similar applanation points, nevertheless, due to its visoelastic nature we get two different pressure measurements at the two applanation points. The difference between these two points results in measurement called corneal hysteresis $(\mathrm{CH}) .{ }^{4-9}$

ORA provides other parameters, such as the Goldman correlated intraocular pressure (IOPg) which is the average reading of the two pressure applanation points, corneal compensated intraocular pressure (IOPCC) (which is the IOP reading that is not influenced by corneal properties, such as corneal thickness) and $\mathrm{CH}$. IOPCC remains stable postLASEK procedure hence providing accurate measurement of IOP.

Corneal resistance factor is another parameter provided by the ORA which is dependent on the central corneal thickness (CCT) and IOPg. Corneal resistance factor is an indication of the corneal elastic properties and measurement of its resistance properties. ${ }^{4,10-12}$

Studies have demonstrated the significance of these ORA parameters in the diagnosis and management of ocular diseases. Biomechanical properties can influence IOP reading as well as refractive results. ${ }^{4,5,10,13}$ Low $\mathrm{CH}$ can predict worsening in visual field mapping in glaucoma patients. ${ }^{8}$

Not all factors influencing biomechanical properties are known to date. ${ }^{4,5}$ However, ocular diseases, age, diurnal variations, and local anesthetic agents were found to influence biomechanical properties, mainly $\mathrm{CH}$. Ocular diseases, such as keratoconus, Fuch's endothelial dystrophy and open angle and normal tension glaucoma were found to reduce $\mathrm{CH}$ and CRF values. ${ }^{6,8,10,13,14}$ There is an ongoing debate as to the influence of age on $\mathrm{CH}$, few studies have demonstrated insignificant influence of age on $\mathrm{CH}$ while establishing a tendency to a lower $\mathrm{CH}$ in the elderly. ${ }^{13,15}$ Others demonstrated a reduction in $\mathrm{CH}$ as we age..$^{10,16,17}$

$\mathrm{CH}$ values are stable throughout the day, unlike CCT which demonstrated higher values upon awakening, returning to basic levels an hour later. ${ }^{15-18}$ Local anesthetic agents did not exert any changes in the $\mathrm{CH}$ or IOPg, however, anesthetics did lower IOPCC and CRF. ${ }^{19}$ IOP and biomechanical parameters were similar between the two sexes. ${ }^{10}$
Some studies have demonstrated an interrelationship between biomechanical parameters themselves; $\mathrm{CH}$ was positively related to CCT yet negatively related to IOP. ${ }^{8-13}$

Various operations exerted an effect upon biomechanical properties of the cornea. Cataract surgery resulted in reduced $\mathrm{CH}$ values, ${ }^{20}$ LASIK and LASEK resulted in reduction in all the biomechanical parameters of the cornea, however, the exact changes with relation to the ablation depth was not examined.

The purpose of this study is to examine the influence of the amount of tissue removed during LASEK procedure upon the biomechanical parameters of the corneal as they are examined by ORA.

\section{METHODS}

Compliance with the Helsinki declaration was approved by Asaf Harofe Medical Center in Israel. Data was collected from patient's files that had undergone LASEK at the Hadassah Optimal Medical Center in Haifa between January 2009 and May 2010. All patients were operated by the same surgeon (AB). We reviewed 239 files, but only included in this study patients who had no less than 3 ORA measurement at 3 different time points: preoperative, early postoperative period (within 3 months of the operation) and late postoperative period (more than 3 months postoperatively). One hundred and forty one patients were excluded due to lack of data. One eye of each of the 98 remaining patients were included in the study. The right eye was selected for randomization purposes for the initial 49 patients and the left eye for the latter 49 patients. The patients were fitted into 3 groups according to their refractive error; low myopia ( 0.5 2.99D), moderate myopia (3.00-5.99D) and high myopia (6.00-15.0D).

We used twin t-tests to assess the change in biomechanical properties at 2 different time points. The relationship between 2 quantitative variables was calculated using the Pearson correlation (the closer the value to 1 the stronger the correlation between the variables). To assess the change over time, the difference between 2 groups and the relationship between them for the variables (CH, CRF, IOPCC and IOPg) we used the ANOVA with repeated measures model. One-way ANOVA model was used to compare quantitative variables between the 3 groups. We used a square linear regression analysis stepwise - model to test the relationship between 2 groups that contained quantitative depended and independent variables. Analysis of covariance (ANCOVA) model was used in which the dependant variables are quantitative and the independent variables can be either quantitative and/or categorical. All statistical tests were bi-directional and $p$ values of $5 \%$ or less was considered statistically significant. 


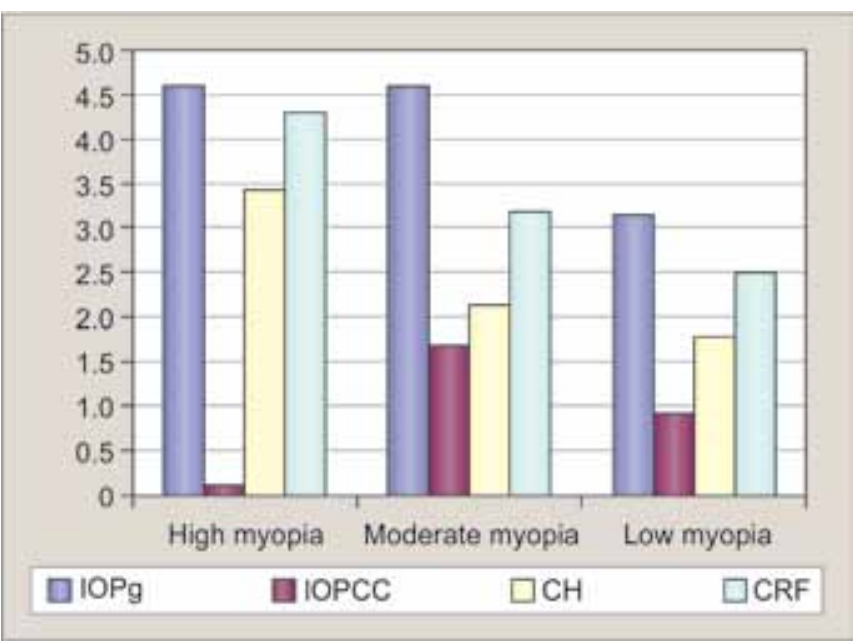

Graph 1: Average change in biomechanical parameters in the 3 groups

\section{RESULTS}

Demographic data is presented in Table 1. Data was collected from 98 patients who underwent LASEK procedure of which 45 are males (45.9\%) and 53 females $(55.1 \%)$. The average age was $29.72 \pm 8.32$ years (19-53 years). Three patients had hyperlipidemia and two had hypothyroidism.

There was statistically significant reduction in the average readings of CCT, IOPg, $\mathrm{CH}$ and $\mathrm{CRF}$ values at the 2 postoperative points when compared to preoperative values ( $\mathrm{p}$ value $<0.001$ ) (Table 2 ).

There was a correlation between the change in biomechanical parameters and IOP to the refractive error postoperatively at the 3 refractive error groups (Table 3, Graph 1).

We tested the relation between the biomechanical parameters to the amount of tissue ablated as tested by TDA and the percentage of tissue ablated as tested by \%TDA/CCT in each of 3 groups. In the low myopia group, there was correlation between the change in $\mathrm{CH}$ and the amount of tissue ablated. $(r=0.4, p=0.012)($ Graph 2$)$ and between the change in $\mathrm{CH}$ and percentage of tissue ablated $(\mathrm{r}=0.39$, $\mathrm{p}=0.013)($ Graph 3). Moreover, there was correlation

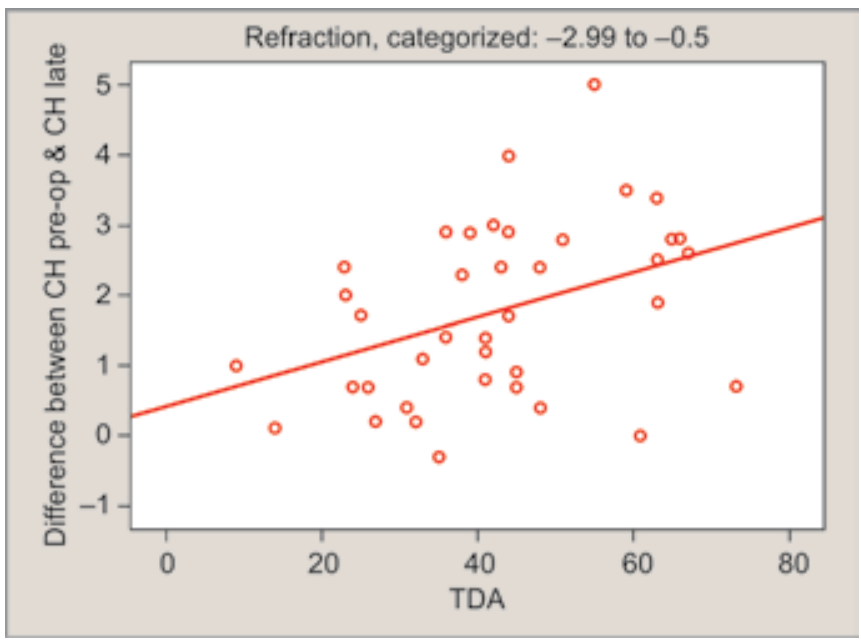

Graph 2: Correlation between TDA and the change in $\mathrm{CH}$ in the late postoperative period (3-12 months)

between the change in CRF and amount of tissue ablated $(r=0.36, p=0.024)$ and between the change in CRF and percentage of tissue ablated $(\mathrm{r}=0.36, \mathrm{p}=0.026)$ (Table 4). No such correlations was demonstrated in the moderate and high myopia groups.

In multivariate analysis to test the effect of variable factors (amount of tissue ablated, percentage of tissue removed, age, sex and refraction) upon the change in biomechanical parameters after LASEK, there was statistically significant relation between the change in $\mathrm{CH}$ and $\mathrm{CRF}$ to age, amount of tissue ablated and percentage of tissue ablated. Hence, a multivariate model was performed for the 2 biomechanical parameters. Since, the dependant variable (the change in parameters between preoperative and postoperative) and the independant variables are quantitative, a stepwise - Linear regression analysis was performed for each of the 2 parameters ( $\mathrm{CH}$ and $\mathrm{CRF})$. In regression analysis of the change in $\mathrm{CH}$ between pre and postoperative there was statistically significant correlation only to age $(p=0.002)$ and no significant relation to the amount ( $p=0.053)$ or percentage of tissue ablated $(p=0.072)$. On testing the change in CRF there

Table 1: Demographic data

\begin{tabular}{lllll}
\hline & All group & Low myopia & Moderate myopia & High myopia \\
& $(n=98)$ & $n=39(39.8 \%)$ & $n=44(44.9 \%)$ & $n=15(15.3 \%)$ \\
\hline Gender (M/F) & $45 / 53$ & $19 / 20$ & $18 / 26$ & $8 / 7$ \\
Mean age $(\mathrm{Y})$ & 29.72 & 32.48 & 28.06 & 29.13 \\
\hline
\end{tabular}

Table 2: Biomechanical parameters, CCT and IOP as measured by ORA in all patients at the 3 time points (preoperative, early postoperative and late postoperative)

\begin{tabular}{|c|c|c|c|c|c|}
\hline & $\begin{array}{l}\text { Preoperative } \\
\text { Mean } \pm S D\end{array}$ & $\begin{array}{l}\text { Early postoperative } \\
\text { Mean } \pm S D\end{array}$ & $p$-value & $\begin{array}{l}\text { Late postoperative } \\
\text { Mean } \pm S D\end{array}$ & $p$-value \\
\hline $\operatorname{CCT}(\mu)$ & $542.84 \pm 33.324$ & & & & \\
\hline IOPg (mm Hg) & $14.287 \pm 3.723$ & $11.52 \pm 3.701$ & 0 & $10.261 \pm 3.027$ & 0 \\
\hline IOPCC (mm Hg) & $14.66 \pm 2.908$ & $15.059 \pm 3.305$ & 0.213 & $13.517 \pm 2.856$ & 0 \\
\hline $\mathrm{CH}(\mathrm{mm} \mathrm{Hg})$ & $10.658 \pm 1.489$ & $8.069 \pm 1.509$ & 0 & $8.465 \pm 1.7605$ & 0 \\
\hline $\mathrm{CRF}(\mathrm{mm} \mathrm{Hg})$ & $10.286 \pm 1.778$ & $7.268 \pm 1.821$ & 0 & $7.203 \pm 1.871$ & 0 \\
\hline
\end{tabular}




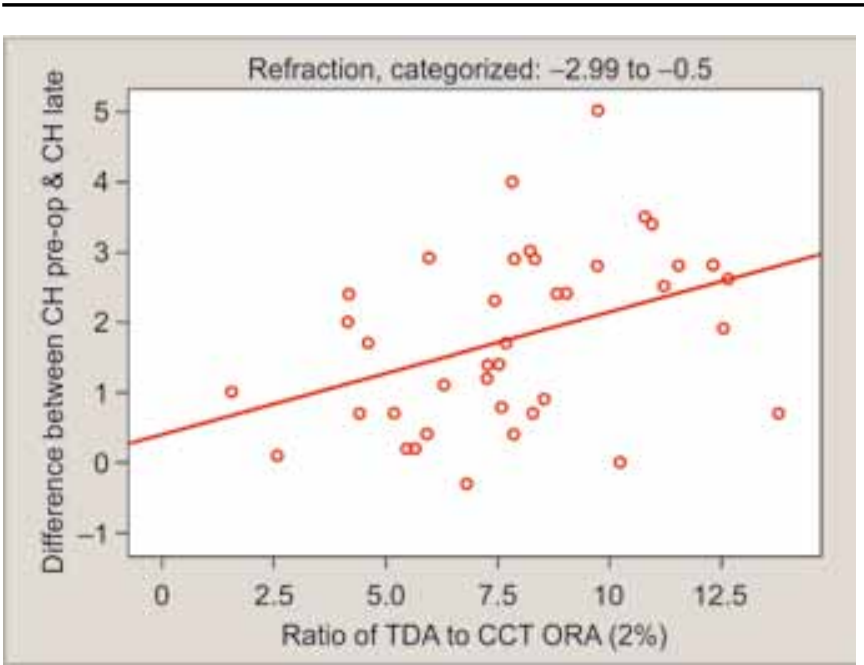

Graph 3: Correlation between the percentage of tissue ablated (CCT/TDA\%) to the change in $\mathrm{CH}$ in the late postoperative period (3-12 months)

was statistically significant relation only to age $(\mathrm{p}=0.003)$. The correlation was not significant to the amount of tissue ablated $(\mathrm{p}=0.093)$ or percentage of tissue ablated $(\mathrm{p}=0.128)$.

We tested the effect of these factors (amount and percentage of tissue ablated, age, sex and refractive error) upon the change in biomechanical parameters in all patients (with no grouping). There was correlation between the amount and percentage of tissue ablated to change in $\mathrm{CH}$ and $\mathrm{CRF}$ values (Table 5).
We used one way ANOVA method to test the influence of refractive error upon the change in the biomechanical parameters. There was statistically significant correlation $(p=0.001)$ between the refractive error and change in biomechanical parameters after LASEK (Appendix 1).

In a covariance analysis, there was statistically significant correlation between age and the change in $\mathrm{CH}$ and $\mathrm{CRF}$.

\section{DISCUSSION}

In literature, there is an emphasis on the importance of corneal biomechanical properties in the diagnosis and management of ocular diseases. ORA enabled the measurement of these parameters, nevertheless not all parameters are known to date. Studies have tested the effect of different factors upon the biomechanical properties. PRK, LASEK and LASIK were found to exert an effect on corneal biomechanical properties as well as diseases such Keratoconus and Fuch's endothelial dystrophy. Diurnal variation of these parameters has been tested as well. In the current study, we tested the effect of different factors on the change in the biomechanical properties after LASEK. The factors tested are amount and percentage of tissue ablated, age, sex and refractive error.

Data from 98 patients was included in the current study, 52 males $(53.1 \%)$ and 46 females (46.9\%). We demonstrated

Table 3: Average change in biomechanical parameters and IOP in the late postoperative period as compared to preoperative period in the 3 refractive error groups

\begin{tabular}{llll}
\hline & Low myopia & Moderate myopia & High myopia \\
\hline IOPg $(\mathrm{mm} \mathrm{Hg})$ & $3.15 \pm 3.19$ & $4.60 \pm 2.1$ & $4.61 \pm 2.5$ \\
IOPCC $(\mathrm{mm} \mathrm{Hg})$ & $0.91 \pm 3.33$ & $1.69 \pm 2.35$ & $0.11 \pm 1.69$ \\
$\mathrm{CH}(\mathrm{mm} \mathrm{Hg})$ & $1.78 \pm 1.24$ & $2.13 \pm 1.19$ & $3.42 \pm 1.23$ \\
$\mathrm{CRF}(\mathrm{mm} \mathrm{Hg})$ & $2.49 \pm 1.05$ & $3.18 \pm 1.2$ & $4.30 \pm 1.55$ \\
\hline
\end{tabular}

Table 4: Correlation between the amount/percentage of tissue ablated during LASEK procedure to the change in biomechanical parameters in each of the refractive error groups

\begin{tabular}{|c|c|c|c|c|c|c|c|}
\hline & & \multicolumn{2}{|c|}{ Low myopia } & \multicolumn{2}{|c|}{ Moderate myopia } & \multicolumn{2}{|c|}{ High myopia } \\
\hline \multicolumn{2}{|c|}{ Correlations } & $T D A$ & TDA/CCT (\%) & $T D A$ & TDA/CCT (\%) & $T D A$ & TDA/CCT (\%) \\
\hline \multirow{3}{*}{$\begin{array}{l}\text { Difference between } \\
\text { IOPg pre-op and } \\
\text { IOPg late }\end{array}$} & $\begin{array}{l}\text { Pearson } \\
\text { Correlation }\end{array}$ & 0.006 & 0.003 & 0.332 & 0.175 & 0.394 & 0.052 \\
\hline & Sig. (2-tailed) & 0.972 & 0.987 & 0.028 & 0.257 & 0.146 & 0.853 \\
\hline & $\mathrm{N}$ & 39 & 39 & 44 & 44 & 15 & 15 \\
\hline \multirow{3}{*}{$\begin{array}{l}\text { Difference between } \\
\text { IOPCC pre-op and } \\
\text { IOPCC late }\end{array}$} & $\begin{array}{l}\text { Pearson } \\
\text { Correlation }\end{array}$ & -0.187 & -0.193 & 0.280 & 0.133 & 0.191 & -0.098 \\
\hline & Sig. (2-tailed) & 0.254 & 0.239 & 0.065 & 0.390 & 0.495 & 0.729 \\
\hline & $\mathrm{N}$ & 39 & 39 & 44 & 44 & 15 & 15 \\
\hline \multirow{3}{*}{$\begin{array}{l}\text { Difference between } \\
\mathrm{CH} \text { pre-op and } \mathrm{CH} \\
\text { late }\end{array}$} & $\begin{array}{l}\text { Pearson } \\
\text { Correlation }\end{array}$ & 0.397 & 0.394 & -0.017 & 0.042 & 0.424 & 0.193 \\
\hline & Sig. (2-tailed) & 0.012 & 0.013 & 0.911 & 0.785 & 0.116 & 0.491 \\
\hline & $\mathrm{N}$ & 39 & 39 & 44 & 44 & 15 & 15 \\
\hline \multirow{3}{*}{$\begin{array}{l}\text { Difference between } \\
\text { CRF pre-op and } \\
\text { CRF late }\end{array}$} & $\begin{array}{l}\text { Pearson } \\
\text { Correlation }\end{array}$ & 0.361 & 0.355 & 0.141 & 0.100 & 0.460 & 0.145 \\
\hline & Sig. (2-tailed) & 0.024 & 0.026 & 0.362 & 0.520 & 0.084 & 0.607 \\
\hline & $\mathrm{N}$ & 39 & 39 & 44 & 44 & 15 & 15 \\
\hline
\end{tabular}


Table 5: Correlation between the change in biomechanical parameters (between preoperative and late postoperative period) to the amount/percentage of tissue ablated and age

\begin{tabular}{|c|c|c|c|c|}
\hline & & Age & $T D A$ & $\begin{array}{l}\text { Ratio of TDA to } \\
\text { CCT ORA (\%) }\end{array}$ \\
\hline \multirow{2}{*}{$\begin{array}{l}\text { Difference between IOPCC } \\
\text { pre-op and IOPCC late }\end{array}$} & Pearson correlation & 0.106 & -0.002 & -0.053 \\
\hline & Sig. (2-tailed) & 0.300 & 0.982 & 0.605 \\
\hline \multirow{2}{*}{$\begin{array}{l}\text { Difference between } \mathrm{CH} \text { pre-op } \\
\text { and } \mathrm{CH} \text { late }\end{array}$} & Pearson correlation & -0.323 & 0.395 & 0.415 \\
\hline & Sig. (2-tailed) & 0.001 & 0 & 0 \\
\hline \multirow{2}{*}{$\begin{array}{l}\text { Difference between CRF pre-op } \\
\text { and CRF late }\end{array}$} & Pearson correlation & -0.355 & 0.477 & 0.465 \\
\hline & Sig. (2-tailed) & 0 & 0 & 0 \\
\hline
\end{tabular}

a statistically significant reduction in the biomechanical parameters post-LASEK $(\mathrm{p}<0.001)$ except of IOPCC which demonstrated a rise in the early postoperative period. The amount/percentage of tissue ablated for all patients did not affect IOPCC readings ( $p=0.982 / 0.605$ respectively). On the contrary, there was a weak-medium correlation between the change in the other biomechanical parameters to the amount/percentage of tissue ablated. The strongest correlation was the change in CRF (Pearson correlation of $0.47 / 0.48$ respectively). We demonstrated a statistically significant correlation $(\mathrm{p}<0.001)$ between age of the patient and the biomechanical parameters with weak-medium correlation upon the change in $\mathrm{CH}$ and $\mathrm{CRF}$, negative Pearson correlation of $-0.32,-0.36$ respectively; the postoperative change in biomechanical parameters was smaller as age rises.

There was no statistically significant effect of the sex of patients on the change in biomechanical parameters of the cornea ( $p=0.283,0.226,0.394,0.776$ for IOPg, IOPCC, CH, and CRF respectively). We grouped patients into 3 refractive error groups (spherical equivalent) and used the One Way Anova model to demonstrate a statistically significant correlation to IOPg ( $<<0.05), \mathrm{CH}$ and CRF $(\mathrm{p}<0.001)$.

In another statistical model, we tested for each biomechanical parameter the factors that influence its postoperative change. For this purpose, we used the analysis of covariance model. There was a statistically significant correlation between the change in IOPg and the amount/percentage of tissue ablated $(p<0.05)$, statistically significant correlation between the change in $\mathrm{CH}$ to age and refractive error $(p<0.05)$ and statistically significant correlation between the change in CRF to age $(\mathrm{p}<0.005)$.

We grouped patients into 3 refractive error groups and tested the influence of the factors upon the change in biomechanical parameters in each group using the Pearson Correlation. There was statistically significant correlation $(\mathrm{p}<0.05)$ in the low myopia group $(-0.5$ to -2.99$)$ between the change in $\mathrm{CH}$ and $\mathrm{CRF}$ to amount/percentage of tissue ablated. The moderate myopia group $(-3.00$ to -5.99$)$ demon-strated a statistically significant correlation $(\mathrm{p}<0.05)$ between the change in IOPg to the amount of tissue ablated. No correlation was found in the high myopia group $(-6.00$ to -15.0$)$ between the change in biomechanical parameters to the amount/percentage of tissue ablated.

Multivariate analysis was used to test for correlation between the change in biomechanical parameters to all the variants (age, sex, amount/percentage of tissue ablated and refraction) in each group. Only in the low myopia group could such a correlation be demonstrated between $\mathrm{CH}$ and $\mathrm{CRF}$ to the amount/percentage of tissue ablated and age. The multivariant model demonstrated a statistically significant correlation between the change in $\mathrm{CH}$ and $\mathrm{CRF}$ to age alone (no relation to amount/percentage of tissue ablated).

All correlations in this study were weak to medium. Previous studies did not demonstrate correlation or influence between amount/percentage of tissue ablated upon the biomechanical properties of the cornea. In our study on 98 patients we found a statistically significant correlation, however this correlation was weak in most cases. Still, there seems to be an inverse correlation to age: the more advanced the age of the patient, the less change we see in the biomechanical parameters of the cornea postoperatively except for IOPCC.

Corneal ectasia is a known complication of LASEK and other refractive procedures. Not all factors leading to ectasia are known. Some known factors are thin cornea, high refractive error, and thick flaps. In the current study, 2 patients had ectasia (2\%), for both patients the CCT was within normal limits $(565 \mu \mathrm{m}$ and $583 \mu \mathrm{m})$, the amount of tissue ablated intraoperatively was 90 and $55 \mu \mathrm{m}$ respectively achieving a postoperative corneal thickness of $510 \mu \mathrm{m}$ and $493 \mu \mathrm{m}$ respectively. Certain reports have described the development of corneal ectasia without the presence of one of the well known risk factors as in the 2 cases described here. Consequently, there is need for further studies to try and define these factors.

In addition to corneal erosion, 6 patients (11.1\%) complained of pain, $6(6.1 \%)$ complained of blurring of vision and $5(5.1 \%)$ complained of dry eyes. Two patients were diagnosed with haze +1 . These symptoms disappeared within weeks to months postoperatively in most cases.

In conclusion, we demonstrated a reduction in the biomechanical properties, except for IOPCC, of the cornea in 
two follow-up points after LASEK procedures. There was a weak to medium correlation which is still statistically significant for different factors upon the change in biomechanical parameters. While sex did not exert any influence, the factors of age, amount/percentage of tissue ablated and refractive error all influence changes in biomechanical parameters. On grouping patients into three groups according to the refractive error we demonstrated similar findings only in the low myopia group. It seems that the higher the refractive error, the less effect age and amount of tissue ablated have on the change in biomechanical properties of the cornea.

\section{REFERENCES}

1. Fong CS. Refractive surgery: the future of perfect vision. Singapore MED J 2007;48(8):709-718.

2. Samuel Chao-Ming Huang, Hung-Chi Jesse Chen. Overview of laser refractive surgery. Chang Gung Med J 31(3):237-254.

3. Mujtaba A, Qazi, Anderson JP, Mahmoud AM, Yoon EY, Roberts CJ, Pepose JS. Postoperative changes in intraocular pressure and corneal biomechanical metrics, Laser in sito keratomileusis versus laser assisted subepithelial keratectomy. J Cataract Refrac Surg 2009 Oct;35:1774-1788.

4. Shah S, Laiquzzaman M, Yeung I, Pan X, Roberts C. The use of ocular response analyzer to determine corneal hysteresis in eyes before and after excimer laser refractive surgery, Contact lens and anterior eye 2009;32:123-128.

5. Laiquzzaman M, Tambe K, Shah S. Comparison of biomechanical parameters in penetrating keratoplasty and normal eyes using the ocular response analyzer. Clinical and Experimental Ophthalmology 2010;38:758-763.

6. Luce DA. Determining in vivo biomechanical properties of the cornea with an ocular response analyzer. J Cataract Refract Surg 2005;31:156-162.

7. Gatinel D, Chaabouni S, Adam PA, Munck J, OD, Puech M, Hoang-Xuan T. Corneal hysteresis, resistance factor, topography, and pachymetry after corneal lamellar flap. J Refract Surg 2007 Jan 23.

8. Kotecha A. What biomechanical properties of the cornea are Relevant for the Clinician? Surv Ophthalmol 2007.
9. Shah S, Laiquzzaman M, Cunliffe I, Mantry S. The use of the Reichert ocular response analyzer to establish the relationship between ocular hysteresis, corneal resistance factor and central corneal thickness in normal eyes. Contact Lens and Anterior Eye 2006;29:257-262.

10. Ortiz D, Pinero D, Shabayek MH. Arnalich-Montiel F, Alio JL. Corneal biomechanical properties in normal, post-laser in situ keratomileusis, and keratoconic eyes. J Cataract Refract Surg 2007 Aug;33:1371-1375.

11. Kynigopoulos M, Schlote T, Kotecha A, Tzamalis A, Pajic B, Haefliger I. Repeatability of intraocular pressure and corneal biomechanical properties measurements by the ocular response analyzer. Klin Monatsbl Augenheilkd 2008;225:357-360.

12. Medeiros FA, Weinreb RN. Evaluation of the influence of corneal biomechanical properties on intraocular pressure measurements using the ocular response analyzer. J Glaucoma 2006;15:364-370.

13. Kamiya K, Hagishima M, Fujimura F, Shimizu K. Factors affecting corneal hysteresis in normal eyes: graefe's archive for clinical and experimental ophthalmology 2007;246(10):1491-1494.

14. Kirwan C, O'keefe M, Lanigan B. Corneal hysteresis and intraocular pressure measurement in children using the richert ocular response analyzer. Am J Ophthalmol 2006;142:990- 992.

15. Kirwanm C, O'Keefe M. Corneal hysteresis using the Reichert ocular response analyzer: findings pre- and post-LASIK and LASEK. Acta Ophthalmol 2008;86:215-218.

16. Kida T, KK Liu J, Weinreb RN. Effects of aging on corneal biomechanical properties and their impact on 24-hour measurement of intraocular pressure. American J Ophthalmol 2008 Oct;146(4):567-572.

17. Laiquzzaman M, Bhojwani R, Cunliffe I, Shah S. Diurnal variation of ocular hysteresis in normal subjects: relevance in clinical context. Clinical and Experimental Ophthalmology 2006;34:114-118.

18. Kida T, Liu JHK, Weinreb RN. Effect of 24-hour corneal biomechanical changes on intraocular pressure measurement. IOVS 2006 Oct;47(10):4422-4426.

19. Ehongo A, De Maertelaer V, Pourjavan S. Effect of topical corneal anaesthesia on ocular response analyzer parameters: pilot study. Int Ophthalmol, 2008;29(5):325-328.

20. Hager A, Loge K, Fullhas M, Schroeder B, Grobherr M, Wiegand W. Changes in corneal hysteresis after clear corneal cataract surgery. American J Ophthalmol 2007 Sep;144(3): 341-346. 